\title{
SUBDIFFERENTIAL ROLLE'S AND MEAN VALUE INEQUALITY THEOREMS
}

\author{
D. Azagra and R. Deville
}

In this note we give a subdifferential mean value inequality for every continuous Gâteaux subdifferentiable function $f$ in a Banach space which only requires a bound for one but not necessarily all of the subgradients of $f$ at every point of its domain. We also give a subdifferential approximate Rolle's theorem stating that if a subdifferentiable function oscillates between $-\varepsilon$ and $\varepsilon$ on the boundary of the unit ball then there exists a subgradient of the function at an interior point of the ball which has norm less than or equal to $2 \varepsilon$.

\section{INTRODUCTION}

Let $X$ be a Banach space and let $\mathcal{U}$ be an open convex subset of $X$. A function $f: \mathcal{U} \longrightarrow \mathbb{R}$ is said to be Fréchet subdifferentiable at a point $x \in \mathcal{U}$ provided there exists $p \in X^{*}$ such that

$$
\liminf _{h \rightarrow 0} \frac{f(x+h)-f(x)-\langle p, h\rangle}{\|h\|} \geqslant 0
$$

and the subdifferential set of $f$ at the point $x$ is defined by

$$
D^{-} f(x)=\left\{p \in X^{*} \mid \liminf _{|h| \rightarrow 0} \frac{f(x+h)-f(x)-\langle p, h\rangle}{\|h\|} \geqslant 0\right\} .
$$

In the same way $f$ is said to be Fréchet superdifferentiable at $x$ whenever there exists $p \in X^{*}$ such that

$$
\limsup _{h \rightarrow 0} \frac{f(x+h)-f(x)-\langle p, h\rangle}{\|h\|} \leqslant 0,
$$

and the superdifferential set of $f$ at $x$ is defined by

$$
D^{+} f(x)=\left\{p \in X^{*} \mid \limsup _{|h| \rightarrow 0} \frac{f(x+h)-f(x)-\langle p, h\rangle}{\|h\|} \leqslant 0\right\} .
$$

Received 21st November, 1996

Supported in part by DGICYT PB 93/0452.

Copyright Clearance Centre, Inc. Serial-fee code: 0004-9729/97 \$A2.00+0.00. 
The function $f$ is said to be Gâteaux subdifferentiable at $x$ provided there exists $p \in X^{*}$ such that for every $h \in X$

$$
\liminf _{t \rightarrow 0} \frac{f(x+t h)-f(x)-\langle p, t h\rangle}{\|t h\|} \geqslant 0
$$

and the Gâteaux subdifferential set of $f$ at the point $x$ is defined by

$$
D_{G}^{-} f(x)=\left\{p \in X^{*} \mid \forall h \in S_{X}, \liminf _{|t| \rightarrow 0} \frac{f(x+t h)-f(x)-\langle p, t h\rangle}{\|t h\|} \geqslant 0\right\} .
$$

Gâteaux superdifferentiability is defined in a similar way. A function $f$ is said to be (Fréchet or Gâteaux) subdifferentiable (respectively, superdifferentiable) on a set $\mathcal{U}$ provided that it is subdifferentiable (respectively, superdifferentiable) at each point $x$ in $\mathcal{U}$. A function $f$ is (Fréchet or Gâteaux) differentiable at $x$ if and only if it is both subdifferentiable and superdifferentiable at $x$, and in this case we have $\{d f(x)\}=$ $D^{-} f(x)=D^{+} f(x)$. On the other hand it is clear that $D^{-} f(x) \subset D_{G}^{-} f(x)$, so that every Fréchet subdifferentiable function is also Gâteaux subdifferentiable.

In this note we give a subdifferential mean value inequality for every continuous Gâteaux subdifferentiable function $f$ which only requires a bound for one but not necessarily all of the subgradients of $f$ at every point $x \in \mathcal{U}$. That is, if for every $x \in \mathcal{U}$ there exists $p \in D_{G}^{-} f(x)$ such that $\|p\| \leqslant M$, then

$$
|f(x)-f(y)| \leqslant M\|x-y\|
$$

for all $x, y \in \mathcal{U}$. From this we can deduce that if a subdifferentiable function $f: \mathcal{U} \rightarrow \mathbb{R}$ satisfies $0 \in D^{-} f(x)$ for all $x \in \mathcal{U}$ then $f$ is necessarily constant. This result cannot be deduced from other subdifferential mean value inequalities like those in [4] or [1].

Moreover it is proved that if $f: \mathcal{U} \longrightarrow \mathbb{R}$ is a Gâteaux subdifferentiable function, $x, y \in \mathcal{U}$ and $M \geqslant 0$ is such that for every $t \in[0,1]$ there exists $p \in D_{G}^{-} f(t x+(1-t) y)$ with $\|p\| \leqslant M$, then $|f(x)-f(y)| \leqslant M\|x-y\|$.

On the other hand we also give a subdifferential approximate Rolle's theorem. Let us recall that Rolle's theorem in finite dimensional spaces states that for every open connected and bounded subset $\mathcal{U}$ in $\mathbb{R}^{n}$ and every continuous function $f: \overline{\mathcal{U}} \longrightarrow \mathbb{R}$ such that $f$ is differentiable in $\mathcal{U}$ and $f$ is constant on $\partial \mathcal{U}$, there exists an $x$ in $\mathcal{U}$ such that $d f(x)=0$. In [8], Shkarin proved that Rolle's theorem fails in a large class of infinite dimensional Banach spaces, including all super-reflexive and all non-reflexive Banach spaces having a Fréchet differentiable norm. In [2] it is conjectured that Rolle's theorem in infinite dimensional Banach spaces holds if and only if the space does not have a $C^{1}$ bump function and this conjecture is proved to be true within the class of those Banach spaces $X$ for which there exist a Banach space $Y$ with an equivalent norm 
$\|$.$\| whose dual norm \|.\|^{*}$ is locally uniformly rotund (LUR) in $Y^{*}$ and a continuous linear injection $T: X \longrightarrow Y$. This condition is satisfied by every WCG Banach space, every space which can be injected in some $c_{0}(\Gamma)$, and even by every $C(K)$ where $K$ is a scattered compact set with $K^{\left(\omega_{1}\right)}=\emptyset$. An interesting approximate version of Rolle's theorem nevertheless remains true in all Banach spaces, as it is shown in [2]. By an approximate Rolle's theorem we mean that if a differentiable function oscillates between $-\varepsilon$ and $\varepsilon$ on the boundary of the unit ball $B_{X}$ then there exists a point in the interior of the ball in which the differential of the function has norm less than or equal to $\varepsilon$. In this note we prove both Fréchet and Gâteaux subdifferential versions of this result within the class of all Banach spaces having a Fréchet (respectively Gâteaux) differentiable Lipschitz bump function (the second one is quite a large class, as it includes all WCG Banach spaces). That is, if a subdifferentiable function oscillates between $-\varepsilon$ and $\varepsilon$ on the boundary of the unit ball then there exists a point $x$ in the interior of the ball and there exists $p \in D^{-} f(x)$ (respectively, $p \in D_{G}^{-} f(x)$ ) such that $\|p\| \leqslant 2 \varepsilon$. In fact, for a Banach space $X$ having a Fréchet differentiable Lipschitz bump function, it is proved that every bounded continuous function $f: B_{X} \rightarrow \mathbb{R}$ such that $f$ oscillates between $-\varepsilon$ and $\varepsilon$ on the unit sphere satisfies $\inf \left\{\|p\|: p \in D^{-} f(x) \cup D^{+} f(x),\|x\|<1\right\} \leqslant 2 \varepsilon$.

\section{Subdifferential MEAN VALUe inEQUALity THEOREM}

THEOREM 2.1. Let $X$ be a Banach space, let $\mathcal{U}$ an open convex subset of $X$ and let $f: \mathcal{U} \longrightarrow \mathbb{R}$ a continuous Gâteaux subdifferentiable function. Suppose that there exists $M \geqslant 0$ such that for every $x \in \mathcal{U}$ there exists $p \in D_{G}^{-} f(x)$ such that $\|p\| \leqslant M$. Then

$$
|f(x)-f(y)| \leqslant M\|x-y\|
$$

for all $x, y \in \mathcal{U}$.

Proof: Let $x, y \in \mathcal{U}, x \neq y$, and let $\varepsilon>0$. Define $h=y-x$ and

$$
A=\{\alpha \in[0,1] \mid f(x+\alpha h)-f(x) \geqslant-(M+\varepsilon) \alpha\|h\|\} .
$$

We show that $A \neq \emptyset$. Take $p \in D_{G}^{-} f(x)$ such that $\|p\| \leqslant M$. Since

$$
\liminf _{|t| \rightarrow 0} \frac{f(x+t h)-f(x)-\langle p, t h\rangle}{\|t h\|} \geqslant 0
$$

there exists $\delta>0$ such that $f(x+t h)-f(x)-\langle p, t h\rangle \geqslant-\varepsilon|t|\|h\|$ whenever $|t| \leqslant \delta$. Then

$$
\begin{aligned}
f(x+t h)-f(x) \geqslant\langle p, t h\rangle-\varepsilon|f|\|h\| & \geqslant-M|t|\|h\|-\varepsilon|t|\|h\| \\
& =-(M+\varepsilon)|t|\|h\| ;
\end{aligned}
$$


and by taking $t=\delta$ we get $f(x+\delta h)-f(x) \geqslant-(M+\varepsilon) \delta\|h\|$, so that $\delta \in A$.

Let $\beta=\sup A \in(0,1]$. Since $\beta=\sup A$, there exists $\left(\alpha_{n}\right) \subset[0, \beta] \cap A$ such that $\alpha_{n} \nearrow \beta$ and $f\left(x+\alpha_{n} h\right)-f(x) \geqslant-(M+\varepsilon) \alpha_{n}\|h\|$ for all $n \in \mathbb{N}$. By letting $n$ go to infinity and using the continuity of $f$, we get $f(x+\beta h)-f(x)=$ $\lim _{n}\left[f\left(x+\alpha_{n} h\right)-f(x)\right] \geqslant \lim _{n}-(M+\varepsilon) \alpha_{n}\|h\|=-(M+\varepsilon) \beta\|h\|$, that is,

$$
f(x+\beta h)-f(x) \geqslant-(M+\varepsilon) \beta\|h\|,
$$

which means $\beta \in A$.

We now show that $\beta=1$. If $\beta<1$, put $z=x+\beta h$ and choose $p \in D_{G}^{-} f(z)$ such that $\|p\| \leqslant M$. Since

$$
\liminf _{|t| \rightarrow 0} \frac{f(z+t h)-f(z)-\langle p, t h\rangle}{\|t h\|} \geqslant 0
$$

there exists $\delta>0$ such that if $|t| \leqslant \delta$ then $f(z+t h)-f(z)-\langle p, t h\rangle \geqslant-\varepsilon\|t h\|$, and so

$$
f(z+t h)-f(z) \geqslant-(M+\varepsilon)|t|\|h\| \text { if }|t| \leqslant \delta .
$$

From (1) and (2) it follows that

$$
\begin{aligned}
f(x+\beta h+t h) & \geqslant f(x+\beta h)-(M+\varepsilon)|t|\|h\| \geqslant f(x)-(M+\varepsilon) \beta\|h\|-(M+\varepsilon)|t|\|h\| \\
& =f(x)-(M+\varepsilon)(\beta+|t|)\|h\|
\end{aligned}
$$

whenever $|t| \leqslant \delta$. By taking $t=\delta$, we obtain

$$
f(x+(\beta+\delta) h) \geqslant f(x)-(M+\varepsilon)(\beta+\delta)\|h\|,
$$

which implies $\beta+\delta \in A$. This is a contradiction because $\beta+\delta>\beta=\sup A$. Thus, $\beta=1$. By substituting $\beta=1$ in (1) we get $f(x+h)-f(x) \geqslant-(M+\varepsilon)\|h\|$ and since $h=y-x$ this means $f(y)-f(x) \geqslant-(M+\varepsilon)\|y-x\|$. This reasoning proves that for all $x, y \in \mathcal{U}$ and for all $\varepsilon>0$ we have

$$
f(x)-f(y) \leqslant(M+\varepsilon)\|y-x\| .
$$

By interchanging $x$ and $y$ we also get $f(y)-f(x) \leqslant(M+\varepsilon)\|y-x\|$. Therefore $|f(y)-f(x)| \leqslant(M+\varepsilon)\|y-x\|$ for all $x, y \in \mathcal{U}$ and for all $\varepsilon>0$. Finally, by fixing $x, y \in \mathcal{U}$ and letting $\varepsilon \searrow 0$ we have $|f(x)-f(y)| \leqslant M\|x-y\|$, so it is proved that $|f(x)-f(y)| \leqslant M\|x-y\|$ for all $x, y \in \mathcal{U}$.

It should be noted that the preceding reasoning in fact proves the following result, which is a subdifferential mean value inequality somewhat flavoured like the classic one. 
THEOREM 2.2. Let $X$ be a Banach space and let $f: \mathcal{U} \longrightarrow \mathbb{R}$ be a Gâteaux subdifferentiable function. If $x, y \in \mathcal{U}$ and $M \geqslant 0$ is such that for every $t \in[0,1]$ there exists $p \in D_{G}^{-} f(t x+(1-t) y)$ with $\|p\| \leqslant M$, then $|f(x)-f(y)| \leqslant M\|x-y\|$.

COROLlary 2.3. Let $\mathcal{U}$ be an open convex subset in a Banach space $X$, and let $f: \mathcal{U} \longrightarrow \mathbb{R}$ be a continuous Gâteaux subdifferentiable function such that $0 \in D_{G}^{-} f(x)$ for all $x \in \mathcal{U}$. Then $f$ is constant on $\mathcal{U}$.

It is not true that if $f: X \longrightarrow \mathbb{R}$ is continuous and subdifferentiable in a dense subset $D \subset X$ and $0 \in D^{-} f(x)$ for all $x \in D$ then $f$ is constant. Even though $X$ is finitely dimensional and the Lebesgue measure of $X \backslash D$ is zero this is not true, as the following example proves.

EXAMPle 2.4. Let $f:[0,1] \longrightarrow \mathbb{R}$ be the Cantor-Lebesgue function (see its definition in $[3$, p.55], for instance). $f$ is non-decreasing and continuous in $[0,1]$, and $f$ is locally constant in $D=[0,1] \backslash C$, where $C$ is Cantor's set. So $f$ is differentiable in $D$, with $\{0\}=\{d f(x)\}=D_{G}^{-} f(x)$ for all $x \in D$, and yet $f$ is not constant.

However, if $\operatorname{dim} X \geqslant 2$, by using some cardinality reasoning one can easily deduce the following improvement of Theorem 2.1.

Corllary 2.5. Let $X$ be a Banach space with $\operatorname{dim} X \geqslant 2$, and let $\mathcal{U} \subset X$ be an open convex subset. Let $f: \mathcal{U} \longrightarrow \mathbb{R}$ be continuous such that $f$ is Gâteaux subdifferentiable in $\mathcal{U} \backslash C$, where $C$ is a countable subset of $\mathcal{U}$. Suppose that there exists $M \geqslant 0$ such that for all $x \in \mathcal{U} \backslash C$ there exists $p \in D_{G}^{-} f(x)$ with $\|p\| \leqslant M$. Then

$$
|f(x)-f(y)| \leqslant M\|x-y\|
$$

for all $x, y \in \mathcal{U}$.

\section{Subdifferential Approximate Rolle's theorem}

In order to prove the subdifferential approximate Rolle's theorems we shall need three auxiliary results.

We shall use the following formula for the subdifferential of the sum (due to Deville and El Haddad [6]) to prove the strongest version of the theorem in the Fréchet case.

THEOREM 3.1. (Formula for the subdifferential of the sum) Suppose $X$ is a Banach space having a $C^{1}(X)$ Lipschitz bump function. Let $f, g: X \longrightarrow \mathbb{R}$ be such that $f$ is lower semicontinuous and $g$ is uniformly continuous. Then, for every $x_{0} \in X, p \in$ $D^{-}(f+g)\left(x_{0}\right)$ and $\varepsilon>0$, there exist $x_{1}, x_{2} \in X, p_{1} \in D^{-} f\left(x_{1}\right)$ and $p_{2} \in D^{-} g\left(x_{2}\right)$ such that:

(i) $\left\|x_{1}-x_{0}\right\|<\varepsilon$ and $\left\|x_{2}-x_{0}\right\|<\varepsilon$.

(ii) $\left|f\left(x_{1}\right)-f\left(x_{0}\right)\right|<\varepsilon$ and $\left|g\left(x_{2}\right)-g\left(x_{0}\right)\right|<\varepsilon$.

(iii) $\left\|p_{1}+p_{2}-p\right\|<\varepsilon$. 
We shall also need the following Variational Principle, whose proof can be found in [5], Chapter I.

Theorem 3.2. (Variational Principle) Let $X$ be a Banach space which has a Fréchet differentiable Lipschitz bump function (respectively Gâteaux differentiable Lipschitz bump function). Let $F: X \rightarrow \mathbb{R} \cup\{\infty\}$ be a lower semicontinuous function that is bounded below, $F \not \equiv+\infty$. Then, for all $\delta>0$ there exists a bounded Fréchet differentiable (respectively, Gâteaux differentiable) Lipschitz function $\varphi: X \longrightarrow \mathbb{R}$ such that:

1. $F-\varphi$ attains its strong minimum in $X$,

2. $\|\varphi\|_{\infty}=\sup _{x \in X}|\varphi(x)|<\delta$, and $\left\|\varphi^{\prime}\right\|_{\infty}=\sup _{x \in X}\left\|\varphi^{\prime}(x)\right\|<\delta$.

Finally, in order to prove the weaker Gâteaux version of the theorem we shall also use the following version of Ekeland's Variational Principle, whose proof can be found in $[7]$.

Theorem 3.3. (Ekeland's Variational Principle) Let $X$ be a Banach space and let $f: X \rightarrow[-\infty, \infty]$ be a proper upper semicontinuous function which is bounded above. Let $\varepsilon>0$ and $x_{0} \in X$ be such that $f\left(x_{0}\right)>\sup \{f(x): x \in X\}-\varepsilon$. Then for any $\lambda$ with $0<\lambda<1$ there exists a point $z \in \operatorname{Dom}(f)$ such that:
(i) $\quad \lambda\left\|z-x_{0}\right\| \leqslant f(z)-f\left(x_{0}\right)$
(ii) $\left\|z-x_{0}\right\|<\varepsilon / \lambda$
(iii) $\quad \lambda\|x-z\|+f(z)>f(x)$ whenever $x \neq z$.

Now let us start with the Fréchet subdifferential approximate Rolle's theorem. Its statement is stronger and the proof is simpler than in the Gâteaux case thanks to the formula for the subdifferential of the sum. Hereafter the set $\{x \in X:\|x\| \leqslant R\}$ is denoted by $B(0, R)$, while $S(0, R)$ stands for $\{x \in X:\|x\|=R\}$.

Theorem 3.4. Let $X$ be a Banach space which has a $C^{1}(X)$ Lipschitz bump function, let $B=B(0, R), S=S(0, R)$ and let $f: B \longrightarrow \mathbb{R}$ be a bounded continuous function such that $f(S) \subset[-\varepsilon, \varepsilon]$. Then:

(i) If $\sup f(B)>\sup f(S)$ then for each $\alpha>0$ there exist $x \in \operatorname{int}(B)$ and $p \in D^{+} f(x)$ such that $\|p\|<\alpha$.

(ii) If inf $f(B)<\inf f(S)$ then for each $\alpha>0$ there exist $x \in \operatorname{int}(B)$ and $p \in D^{-} f(x)$ such that $\|p\|<\alpha$.

(iii) If $f(B) \subseteq f(S)$ then for each $\alpha>0$ there exist $x_{1}, x_{2} \in \operatorname{int}(B)$ and $p_{1} \in D^{+} f\left(x_{1}\right), p_{2} \in D^{-} f\left(x_{2}\right)$ such that $\left\|p_{1}\right\|,\left\|p_{2}\right\|<2 \varepsilon / R+\alpha$.

PROOF:

CASE (I). Let $\eta=\sup f(B)-\sup f(S)>0$, and consider $F(x)=f(x)$ if $x \in$ $B, F(x)=-\infty$ otherwise. Since $F$ is upper semicontinuous and bounded above, 
the Variational Principle provide us with a $C^{1}(X)$ function $g$ such that $\|g\|<\eta / 3$, $\left\|g^{\prime}\right\|<\alpha$ and $F+g$ attains its maximum at a point $x \in B$. Moreover $x \in \operatorname{int}(B)$ : otherwise, by taking $a$ such that $f(a)>\sup f(B)-\eta / 3$ we would get

$$
\sup f(B)-2 \eta / 3<F(a)+g(a) \leqslant F(x)+g(x) \leqslant \sup f(S)+\eta / 3,
$$

which is a contradiction. Therefore $x \in \operatorname{int}(B)$ and $p=g^{\prime}(x) \in D^{+} f(x)$ satisfies $\|p\|<\alpha$.

CASE (II). The same proof works.

CASE (III). Let us consider the function $\phi(x)=f(x)-(2 \varepsilon+\alpha)\|x\| / R$. This function satisfies the conditions of Case (I) and so there exist $x \in \operatorname{int}(B)$ and $p \in D^{+} \phi(x)$ such that $\|p\|<\alpha$. Now, by the formula for the subdifferential of the sum, there exist $x_{1}, y_{1} \in \operatorname{int}(B)$ and $p_{1}, q_{1}$ with $p_{1} \in D^{+} f\left(x_{1}\right), q_{1} \in D^{+}\left(-(2 \varepsilon+\alpha) / R\left\|y_{1}\right\|\right)$ such that $\left\|p_{1}+q_{1}-p\right\|<\alpha$, which implies

$$
\left\|p_{1}\right\|<\alpha+\left\|q_{1}\right\|+\|p\|<2 \alpha+\left\|q_{1}\right\| .
$$

Let us note that $q \in D^{+}(-\|\|).(v)$ if and only if $-q \in D^{-}(\|\|).(v)$. Moreover, since $\|$.$\| is convex we have \partial\|\cdot\|(v)=D^{-}\|\cdot\|(v)$, so that if $q \in D^{-}\|\cdot\|(v)$ then $q(h) \leqslant$ $\|v+h\|-\|v\| \leqslant\|h\|$ for all $h$, and therefore $\|q\| \leqslant 1$. Taking this into account we can deduce that $\left\|q_{1}\right\|=\left\|-q_{1}\right\| \leqslant(2 \varepsilon+\alpha) / R$ and so $\left\|p_{1}\right\|<2 \alpha+(2 \varepsilon+\alpha) / R$.

In order to find $x_{2}$ and $p_{2}$ it is enough to consider $\phi(x)=f(x)+(2 \varepsilon+\alpha)\|x\| / R$ and the same proof holds using Case (II) instead of (I).

From this result we deduce the following

THEOREM 3.5. Let $\mathcal{U}$ be an open connected bounded set in a Banach space $X$ which has a $C^{1}(X)$ Lipschitz bump function, let $f: \mathcal{U} \longrightarrow \mathbb{R}$ be a bounded continuous function and let $R>0$ and $x_{0} \in \mathcal{U}$ be such that $B\left(x_{0}, R\right) \subseteq \overline{\mathcal{U}}$. Suppose that $f(\partial \mathcal{U}) \subseteq[-\varepsilon, \varepsilon]$. Then:

(i) If $\sup f(\overline{\mathcal{U}})>\sup f(\partial \mathcal{U})$ then for each $\alpha>0$ there exist $x \in \mathcal{U}$ and $p \in D^{+} f(x)$ such that $\|p\|<\alpha$.

(ii) If $\inf f(\overline{\mathcal{U}})<\inf f(\partial \mathcal{U})$ then for each $\alpha>0$ there exist $x \in \mathcal{U}$ and $p \in D^{-} f(x)$ such that $\|p\|<\alpha$.

(iii) If $f(\bar{U}) \subseteq f(\partial \mathcal{U})$ then for each $\alpha>0$ there exist $x_{1}, x_{2} \in \mathcal{U}$ and $p_{1} \in$ $D^{+} f\left(x_{1}\right), p_{2} \in D^{-} f\left(x_{2}\right)$ such that $\left\|p_{1}\right\|,\left\|p_{2}\right\|<2 \varepsilon / R+\alpha$.

In each case, $\inf \left\{\|p\|: p \in D^{-} f(x) \cup D^{+} f(x), x \in \mathcal{U}\right\} \leqslant 2 \varepsilon / R$.

From this we can immediately deduce 
Corollary 3.6. Let $\mathcal{U}$ be an open connected bounded subset of a Banach space $X$ that has a Fréchet differentiable Lipschitz bump function, and let $f: \overline{\mathcal{U}}: \longrightarrow \mathbb{R}$ be continuous and bounded on $\mathcal{U}$. Suppose that $f$ is constant on $\partial \mathcal{U}$. Then

$$
\inf \left\{\|p\|: p \in D^{-} f(x) \cup D^{+} f(x), x \in \mathcal{U}\right\}=0 .
$$

and also

Corollary 3.7. Let $X$ be a Banach space having a Fréchet differentiable Lipschitz bump function and let $f: X \rightarrow \mathbb{R}$ be continuous and bounded on $X$. Then

$$
\inf \left\{\|p\|: p \in D^{-} f(x) \cup D^{+} f(x), x \in X\right\}=0 .
$$

Finally we shall study the subdifferential approximate Rolle's theorem in the Gâteaux case. Here the proof is longer and the statement weaker than in the Fréchet case. If the formula for the subdifferential of the sum were true in the Gâteaux case within the class of those Banach spaces having a Gâteaux differentiable and Lipschitz bump function, the proof of Theorem 3.4 would also work in this case yielding an improvement in the statement of theorem 3.8 and its corollaries. We do not know whether such a formula is true or not within that class of Banach spaces.

THEOREM 3.8. Let $X$ be a Banach space which has a Gâteaux differentiable Lipschitz bump function and let $R, \varepsilon>0$. Let $f: B(0, R) \rightarrow \mathbb{R}$ be a continuous bounded function on $B(0, R)$ and suppose that $f$ is Gâteaux subdifferentiable in int $B(0, R)$ and $f(S(0, R)) \subseteq[-\varepsilon,+\varepsilon]$. Then there exist $x_{\varepsilon} \in$ int $B(0, R)$ and $p \in D_{G}^{-} f\left(x_{\varepsilon}\right)$ such that $\|p\| \leqslant 2 \varepsilon / R$.

PROOF: Let us suppose first that $\varepsilon<2 R$. We shall consider three cases.

CASE I. $f(B(0, R)) \subseteq f(S(0, R)) \subseteq[-\varepsilon, \varepsilon]$. Suppose first that $f(0)>-\varepsilon$. Let $\lambda=2 \varepsilon / R$. Since $f(0)>\sup \{f(x): x \in B(0, R)\}-2 \varepsilon$, Ekeland's Variational Principle gives us $x_{1} \in B(0, R)$ such that

$$
\begin{aligned}
\text { (i) } & \lambda\left\|x_{1}\right\| \leqslant f\left(x_{1}\right)-f(0) \\
\text { (ii) } & \left\|x_{1}\right\|<2 \varepsilon / \lambda \\
\text { (iii) } & \lambda\left\|x-x_{1}\right\|+f\left(x_{1}\right)>f(x) \text { whenever } x \neq x_{1},
\end{aligned}
$$

so that $x_{1} \in \operatorname{int} B(0, R)$ and, by taking $p \in D_{G}^{-} f\left(x_{1}\right)$, (iii) implies that $\|p\| \leqslant 2 \varepsilon / R$. Indeed, for every $h$ with $\|h\|=1$ we have

$$
\frac{f\left(x_{1}+t h\right)-f\left(x_{1}\right)}{|t|}<\frac{2 \varepsilon}{R}
$$

for every $t$, and also, since $p \in D_{G}^{-} f\left(x_{1}\right)$,

$$
\liminf _{t \rightarrow 0} \frac{f\left(x_{1}+t h\right)-f\left(x_{1}\right)-t p(h)}{|t|} \geqslant 0
$$


or equivalently

$$
\limsup _{t \rightarrow 0} \frac{-f\left(x_{1}+t h\right)+f\left(x_{1}\right)+t p(h)}{|t|} \leqslant 0,
$$

and therefore

$$
\begin{aligned}
|p(h)| & =\limsup _{t \rightarrow 0} \frac{p(t h)}{|t|}=\limsup _{t \rightarrow 0} \frac{f\left(x_{1}+t h\right)-f\left(x_{1}\right)-f\left(x_{1}+t h\right)+f\left(x_{1}\right)+p(t h)}{|t|} \\
& \leqslant \limsup _{t \rightarrow 0} \frac{f\left(x_{1}+t h\right)-f\left(x_{1}\right)}{|t|}+\limsup _{t \rightarrow 0} \frac{-f\left(x_{1}+t h\right)+f\left(x_{1}\right)+p(t h)}{|t|} \\
& \leqslant \limsup _{t \rightarrow 0} \frac{f\left(x_{1}+t h\right)-f\left(x_{1}\right)}{|t|} \leqslant \frac{2 \varepsilon}{R} .
\end{aligned}
$$

This proves that $\|p\| \leqslant 2 \varepsilon / R$. Now suppose that $f(0)=-\varepsilon$ and pick $p \in D_{G}^{-} f(0)$. We may suppose that $\|p\|>2 \varepsilon / R$ since we would have finished otherwise. Then there exists $h$ with $\|h\|=1$ such that $p(h)>2 \varepsilon / R$. As

$$
\liminf _{t \rightarrow 0} \frac{f(t h)-f(0)-t p(h)}{|t|} \geqslant 0
$$

and $f(0)=-\varepsilon$, there exists $\delta>0$ such that

$$
\frac{f(\delta h)+\varepsilon-\delta p(h)}{\delta}>\frac{2 \varepsilon}{R}-p(h),
$$

which implies $f(\delta h)+\varepsilon>(2 \varepsilon \delta) / R$. Hence $f(\delta h)>\sup f(B(0, R))-2 \varepsilon$. On taking $\lambda=2 \varepsilon / R$ we can again use Ekeland's Variational Principle to get $x_{1} \in B(0, R)$ such that:

(i) $\lambda\left\|x_{1}-\delta h\right\| \leqslant f\left(x_{1}\right)-f(\delta h)$

(ii) $\left\|x_{1}-\delta h\right\|<\varepsilon / \lambda$

(iii) $\lambda\left\|x-x_{1}\right\|+f\left(x_{1}\right)>f(x)$ whenever $x \neq x_{1}$.

From (i) and since $f(\delta h)+\varepsilon>(2 \varepsilon \delta) / R$ we get

$$
\left\|x_{1}-\delta h\right\| \leqslant \frac{f\left(x_{1}\right)-f(\delta h)}{2 \varepsilon / R} \leqslant \frac{\varepsilon-f(\delta h)}{2 \varepsilon / R}<\frac{2 \varepsilon-(2 \varepsilon \delta) / R}{2 \varepsilon / R}=R-\delta
$$

which implies $\left\|x_{1}\right\| \leqslant\left\|x_{1}-\delta h\right\|+\delta<R-\delta+\delta=R$ and so $\left\|x_{1}\right\|<R$. Now, since $f$ is Gâteaux subdifferentiable at $x_{1}$, the same calculations as above prove that (iii) implies $\|p\| \leqslant 2 \varepsilon / R$ for any $p \in D_{G}^{-} f\left(x_{1}\right)$.

CASE II. $\sup f(B(0, R))>\sup f(S(0, R))$. Choose $x_{0}$ such that $\sup f(S(0, R))<$ $f\left(x_{0}\right)$, and let $\alpha, \lambda$ be such that $0<\alpha<f\left(x_{0}\right)-\sup f(S(0, R)), \alpha \leqslant 2 \varepsilon / R$ and $0<\lambda<\alpha /(R+1)$. From Ekeland's Variational Principle it follows that there exists $x_{1} \in \operatorname{int} B(0, R)$ such that

$$
f(x)<f\left(x_{1}\right)+\lambda\left\|x-x_{1}\right\|
$$

for every $x \neq x_{1}$, and we already know that this implies that $\|p\| \leqslant \lambda<\alpha$ for any $p \in D_{G}^{-} f\left(x_{1}\right)$. 
CASE III. inf $f(B(0, R))<\inf f(S(0, R))$. This is the only case in which we shall use the smooth variational principle. Let $\eta=\inf f(S(0, R))-\inf f(B(0, R))>0$, let $\alpha>0$ be such that $\alpha \leqslant 2 \varepsilon / R$ and consider $F: X \rightarrow \mathbb{R} \cup\{\infty\}$ defined by $F(x)=f(x)$ if $x \in B(0, R)$ and $F(x)=+\infty$ otherwise. From the smooth variational principle it follows that there exists a bounded Gâteaux differentiable Lipschitz function $\varphi: X \rightarrow \mathbb{R}$ such that $\|\varphi\|_{\infty}<\eta / 3,\left\|\varphi^{\prime}\right\|_{\infty}<\alpha$ and $F-\varphi$ attains its minimum at a point $x_{0} \in B(0, R)$. Moreover we must have $x_{0} \in \operatorname{int} B(0, R)$ : otherwise, by taking $a$ such that $f(a)<\inf f(B(0, R))+\eta / 3$ we would have

$$
\inf f(B(0, R))+2 \eta / 3>F(a)-\varphi(a) \geqslant F\left(x_{0}\right)-\varphi\left(x_{0}\right) \geqslant \inf f(S(0, R))-\eta / 3,
$$

which is a contradiction. It is easy to check that the sum $g+h$ of two subdifferentiable functions $g$ and $h$ is subdifferentiable, and

$$
D_{G}^{-} g(x)+D_{G}^{-} h(x) \subseteq D_{G}^{-}(g+h)(x)
$$

and it is obvious that if a function $g$ attains a minimum at $x$ then $g$ is subdifferentiable at $x$ and $0 \in D_{G}^{-} g(x)$. Taking this into account we can deduce

$$
0+\varphi^{\prime}\left(x_{0}\right) \in D_{G}^{-}(F-\varphi)\left(x_{0}\right)+D_{G}^{-} \varphi\left(x_{0}\right) \subseteq D_{G}^{-} F\left(x_{0}\right)=D_{G}^{-} f\left(x_{0}\right)
$$

so that $p=\varphi^{\prime}\left(x_{0}\right)$ satisfies $p \in D_{G}^{-} f\left(x_{0}\right)$ and $\|p\|<\alpha \leqslant 2 \varepsilon / R$.

Finally, consider the case in which $\varepsilon \geqslant 2 R$. Taking into account that $p \in D_{G}^{-} f(x)$ if and only if $r p \in D_{G}^{-}(r f)(x)$ for every $r>0$ and considering $g=\varepsilon^{\prime} f / \varepsilon$, where $\varepsilon^{\prime}<2 R$, we can conclude (by applying the preceding reasoning to $g$ ) that there exist $x$ in the interior of the ball and a subgradient $p \in D_{G}^{-} f(x)$ such that $\|p\| \leqslant 2 \varepsilon / R$. $]$

REMARK 3.9. Note that we have only used the smooth variational principle in the proof corresponding to the case inf $f(B(0, R))<\inf f(S(0, R))$. Note also that in the first case we only used that $f(B(0, R)) \subseteq[-\varepsilon, \varepsilon]$. Thus it is clear that for any Banach space $X$ and any Gâteaux subdifferentiable continuous bounded function $f: B_{X}(0, R) \longrightarrow \mathbb{R}$ which is Gâteaux subdifferentiable in the interior of the ball and satisfies $f \geqslant-\varepsilon$ and $f_{\mid S(0, R)} \leqslant \varepsilon$, there exists a point $x$ in the interior of the ball and a subgradient $p \in D_{G}^{-} f(x)$ such that $\|p\| \leqslant 2 \varepsilon / R$.

From the preceding theorem we deduce the more general:

THEOREM 3.10. Let $\mathcal{U}$ be an open connected bounded set in a Banach space $X$ that has a Gâteaux differentiable Lipschitz bump function. Let $f: \overline{\mathcal{U}} \longrightarrow \mathbb{R}$ be continuous and bounded, Gâteaux subdifferentiable in $\mathcal{U}$. Let $R>0$ and $x_{0} \in \mathcal{U}$ be such that dist $\left(x_{0}, \partial \mathcal{U}\right)=R$. Suppose that $f(\partial \mathcal{U}) \subset[-\varepsilon, \varepsilon]$. Then there exist $x_{\varepsilon} \in \mathcal{U}$ and $p \in D_{G}^{-} f\left(x_{\varepsilon}\right)$ such that $\|p\| \leqslant 2 \varepsilon / R$.

Corollary 3.11. Let $\mathcal{U}$ be an open connected bounded subset of a Banach space $X$ that has a Gâteaux differentiable Lipschitz bump function, and let $f: \overline{\mathcal{U}}: \longrightarrow$ 
$\mathbb{R}$ be continuous, bounded, and Gâteaux subdifferentiable in $\mathcal{U}$. Suppose that $f$ is constant on $\partial \mathcal{U}$. Then

$$
\inf \left\{\|p\|: p \in D_{G}^{-} f(x), x \in \mathcal{U}\right\}=0
$$

Corollary 3.12. Let $X$ be a Banach space having a Gâteaux differentiable Lipschitz bump function and let $f: X \longrightarrow \mathbb{R}$ be continuous, Gâteaux subdifferentiable and bounded on $X$. Then

$$
\inf \left\{\|p\|: p \in D_{G}^{-} f(x), x \in X\right\}=0 .
$$

\section{REFERENCES}

[1] D. Aussel, J.-N. Corvellec and M. Lassonde, 'Mean value property and subdifferential criteria for lower semicontinuous functions', Trans. Amer. Math. Soc. 347 (1995), 4147-4161.

[2] D. Azagra, J. Gómez and J. A. Jaramillo, 'Rolle's Theorem and negligibility of points in infinite dimensional Banach spaces', J. Math. Anal. Appl. (to appear).

[3] D.L. Cohn, Measure theory (Birkhäuser, Boston, 1980).

[4] R. Deville, 'A mean value theorem for the non differentiable mappings', Serdica 21 (1995), $59-66$.

[5] R. Deville, G. Godefroy and V. Zizler, Smoothness and renormings in Banach spaces, Pitman Monographs and Surveys in Pure and Applied Mathematics 64 (Longman Science and Technology, Harlow, 1993).

[6] E.M. El Haddad, Calcul sous-differentiel et solutions de viscosité des équations de Hamilton-Jacobi (Thèse doctorale présentée à l'Université Bordeaux I, 1994).

[7] R.R. Phelps, Convex functions, monotone operators and differentiability, Lecture Notes in Mathematics 1364 (Springer-Verlag, Berlin, Heidelberg, New York, 1989).

[8] S.A. Shkarin, 'On Rolle's theorem in infinite-dimensional Banach spaces', Trans. Matematicheskie Zametki 51 (1992), 128-136.

Dpto. de Análisis Matemático

Facultad de Matemáticas

Universidad Complutense de Madrid

28040 Madrid

Spain

e-mail: daniel@sunam1.mat.ucm.es
Département de Mathématiques Pures et Appliquées

Universiti: Bordeaux I

351 , cours de la Libération

33405 Talence Cedex

France

e-mail: deville@math.u-bordeaux.fr 